\title{
Numerical simulation of flow in the wet scrubber for desulfurization
}

\author{
Jan Novosád ${ }^{1, \mathrm{a}}$ and Tomáš Vít ${ }^{1}$
}

${ }^{1}$ Department of Power Engineering Equipment, Faculty of Mechanical Engineering, Technical University of Liberec, Studentska 2, 46117, Liberec, Czech Republic

\begin{abstract}
This article deals with numerical simulation of flow and chemical reactions in absorber for desulfurization of flue-gas. The objective of the work is the investigation of effect of different nozzles types and their placement in spray layers. These nozzles distribute lime suspension into flue gas stream. The research includes two types of nozzles and four different arrangements of nozzles and spray layers. Conclusion describes the effect of nozzle types and their arrangements on the suspension concentration in absorber.
\end{abstract}

\section{Introduction}

Coal-fired power plants in the Czech Republic are still a significant source of electricity. They produce a large amount of gases, which are harmful for the environment, especially $\mathrm{NO}_{x}$ and $\mathrm{SO}_{2}$. Amount of pollutants generated during combustion is several times more than the amount prescribed by legislation. To reduce the amount of $\mathrm{SO}_{2}$ in the emissions, it is necessary to install the flue gas cleaning device. The world's most widely used device for flue gas desulfurization process is the wet limestone scrubber. In the scrubber there occur several chemical reactions. The reactants are sulfur dioxide (in flue gas) and an aqueous suspension of finely ground limestone. The products are clean flue gas and calcium sulfate hydrate $\left(\mathrm{CaCO}_{4} \times 2 \cdot \mathrm{H}_{2} \mathrm{O}\right)$, ie. Gypsum. The efficiency of $\mathrm{SO}_{2}$ reduction is high, reaching up to $96 \%$ but with a high excess of the suspension. Desulphurization product gypsum is very useful especially in construction, where fully replace natural gypsum used. Gypsum is used as an ingredient in the manufacture of cement and gypsum.

The process of desulfurization depends on many parameters. Currently there is no mechanism that would allow design the wet scrubber with the warranty of high efficiency of $\mathrm{SO}_{2}$ reduction. Most equipment in the first set is less than the required parameters, which indicate the amount of emissions or operating inefficiently, with high flow rates of suspension.

The aim of our research is to describe the process of desulfurization with respect to individual parameters (arrangement and type of nozzles, design of the drop eliminator etc.), carry a balance of components in the desulfurization reaction and propose a procedure for the numerical simulation of desulphurization process.

The aim of this article is the investigation of the effect of nozzles to desulphurization process.

\section{Numerical simulation}

The research subject is the flow of flue gas through the scrubber. In the scrubber there are situated spray layers with nozzles. The nozzles spraying lime suspension into the gas stream. It is simulated several variants with different types of nozzles and their arrangement. The calculation is steady state with the possibility of interaction between the gas phase (flue gas) and liquid phase (suspension droplets).

\subsection{Geometry and mesh}

The scrubber was divided into separate parts - the lower part, several nozzle layers and the upper part. Mesh consisted of hexahedrons with local refinement in the field of nozzles. Between the parts the boundary condition was applied Interface, in these areas the mesh is not corresponding.

We used nozzles type Double WhirlJet with full and empty cone and type Twin Absorb with full and hollow cone (see Figure 1).
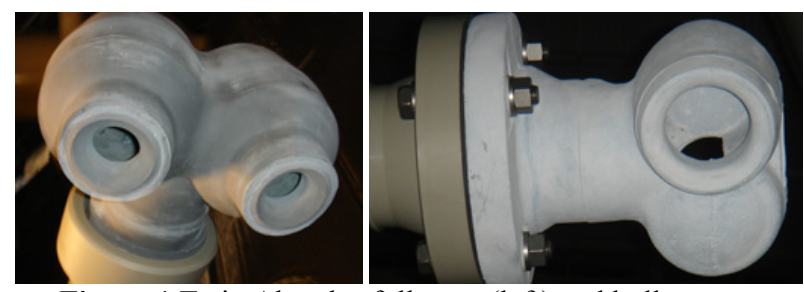

Figure 1.Twin Absorb - full cone (left) and hollow cone.

In the basic configuration (variant I) the nozzles are located in three layers. Double WhirlJet nozzles with full and hollow cone are used. Placement of nozzles is shown in figure 2 .

Variant II has the same configuration of nozzles as var. I. In the third layer there were used WhirlJet

\footnotetext{
${ }^{\mathrm{a}}$ Corresponding author: jan.novosad@tul.cz
} 
nozzles with hollow cone in the middle area and with full cone on the edge of the layer.

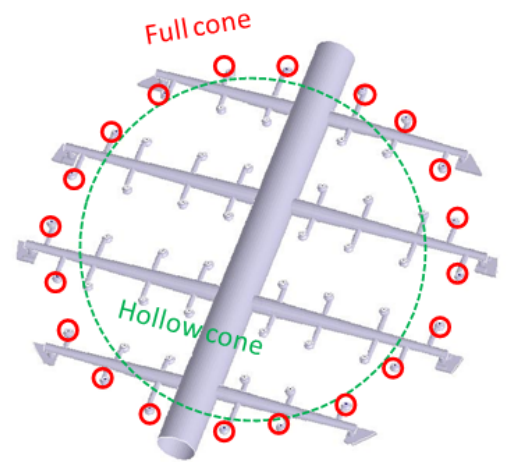

Figure 2. Spray layer - basic placement of nozzles.

Variant III is based on var. II. 4 nozzles were added to the second layer (see figure 3).

Variant IV is based on var. II. A new layer has been added between layer 2 and layer 3. The newly added layer has the same configuration as the layer 2 in variant II. This model consists of 4 layers.

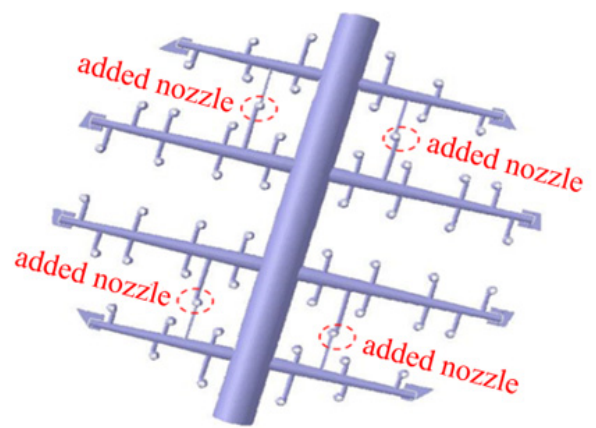

Figure 3.Variant III - Spray layer 2 - added nozzles.

\subsection{Boundary conditions}

Figure 4 shows the boundary conditions used in calculations. Inlet was set as "Mass flow inlet". The flue gas composition is shown in table in figure 4. The exhaust area is set as pressure outlet.

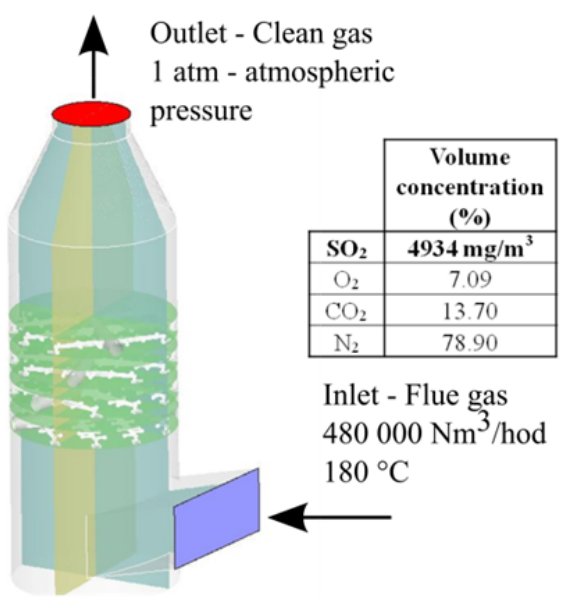

Figure 4.Basic geometry of the absorber.

The flow of suspension through each nozzle is based on the total flow of suspension through one layer of which was $3700 \mathrm{~m}^{3} / \mathrm{h}$.
Planes for displaying results are shown in figure 5 . Planes y7 and y8 are used only for displaying the results of variant IV.

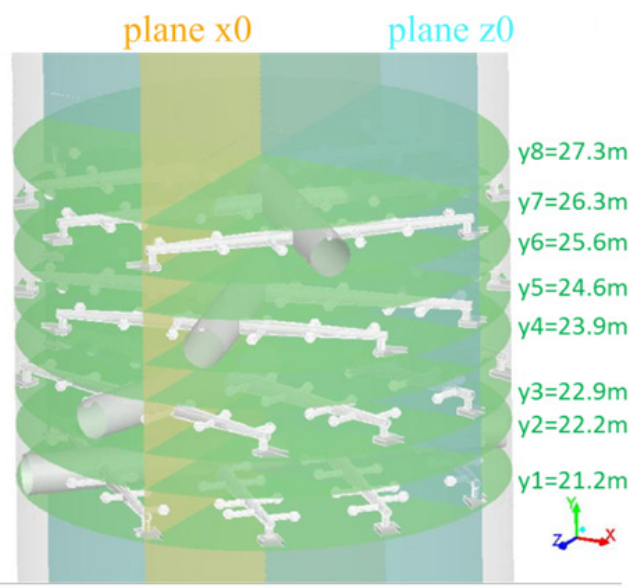

Figure 5. Planes for displaying results.

\subsection{Solution}

For solution was used ANSYS FLUENT 14.5.0 (3D, pressure-based). Flue gas is defined as a mixture of ideal gases with temperature-dependent material properties. Viscous model k- $\varepsilon$ Realizable was used. The suspension particles were modelled as an incompressible fluid with heat and mass transfer between the particles and the gas. The particle density is $1080 \mathrm{~kg} / \mathrm{m}^{3}$. TRAP model was used therefore particles in contact with wall run down the wall, not bounce.

\section{Results}

The kinetics of chemical reactions in a wet scrubber is influenced by many parameters. The most important are the temperature, size of the particles of suspension, the concentration of the reactants in the suspension, the distribution of the reactants in the suspension, the relative speed of the flue gas and particles of suspension and the concentration of the particles of suspension in the reaction zone.

The presented model can relatively accurately describe the distribution of concentration of the sprayed suspension in the reaction zone, a relative velocity between the flue gas and particles of suspension and the residence time of the flue gas in zones with a sufficient concentration of suspension.

In principle, this model can also be used to simulate a process of the individual chemical reactions. To achieve reliable results it is necessary to precisely define the parameters of chemical reactions. The required input data are not freely available and they must be obtained on the basis of precise experiments.

Procedure for quantitative evaluation is based on monitoring the time that the volume part of flue gas remains in the zone with a specific concentration of suspension. It is assumed that for optimum process of chemical reactions it is necessary that the volume part of flue gas remains for a specified time in the zone with a specific threshold concentration of the suspension. Specified time and the threshold concentration of the suspension must be obtained from the experiments. 
Analysis of the average concentration of suspension in the zone in which the flue gas volume part is located can bring some insight.

Figure 6 shows the individual streamlines of flue gas. Each streamline represents a certain volume of flue gas and its predicted path through the wet scrubber. Streamlines are coloured according to the concentration of the suspension at a certain location. In the analyzed model, there can be displayed and evaluated data from approximately 1800 streamlines. This quantity is sufficient for statistical evaluation.

Figure 6 also shows the concentration of suspension along the path of three selected particles. For one of the particles is indicated the average value of concentration of suspension.

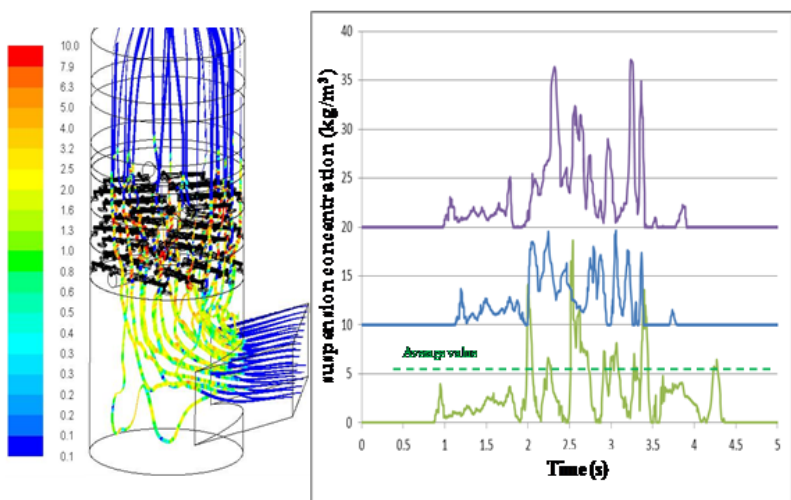

Figure 6. Streamlines coloured by concentration (left) and values of concentration along the path of selected particles.

Figure 7 shows the percentage of volume flow rate with respect to the average value of the suspension in the zone in which the volume of flue gas was located.

Low values of average concentration indicate imperfect placement of nozzles or insufficient amounts of suspension (low flow rate of the suspension through the system). High values of average concentration may indicate unnecessarily high flow rate of suspension. Optimal zone must be defined on the basis of experience or operational or special experiments.

In this case it is possible to see the following trends:

- The initial state is very close to the ideal distribution.

- Changing nozzles type (WhirlJet for Twin Absorb) in the third floor has only minimal effect on the concentration. Small increase of concentration "in the optimum zone" is caused by more intense uplift, which means a longer residence time of smaller particles in the wet scrubber. The advantage of this exchange is to reduce the diameter of the suspension drops which causes an increase of the reaction surface.

- Adding the nozzles into the second layer causes improving parameters of scrubber.

- Adding a fourth layer causes a significant increase of average concentration. This increase may not lead to reduce the amount of $\mathrm{SO}_{2}$ in the flue gas.

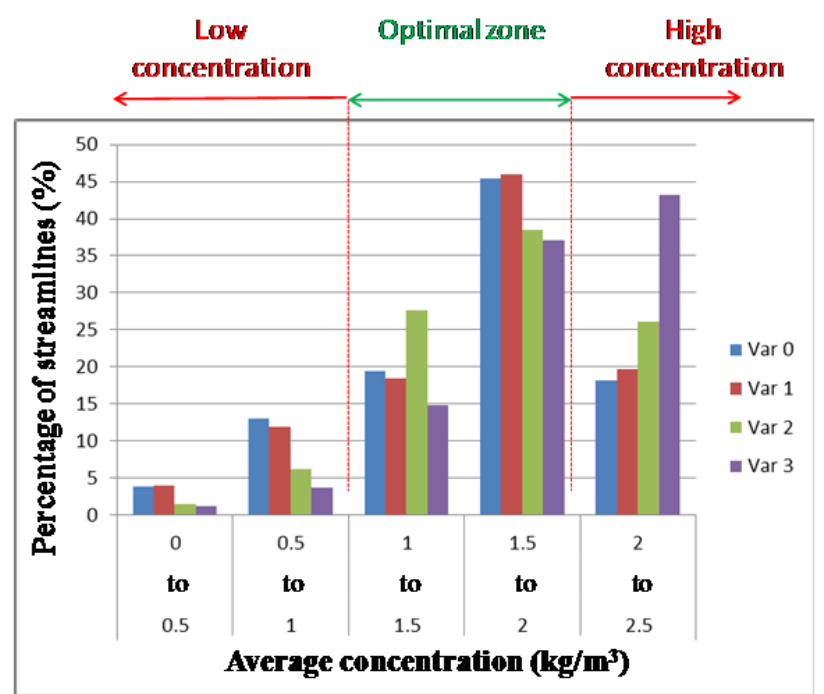

Figure 7. Percentage of streamlines.

Figure 8 shows the concentration of suspension displayed in plane $\mathrm{z} 0$ for all variants.

The concentration of suspension displayed in plane y1-y6 (y8) for all variants are shown in figures 9-12.

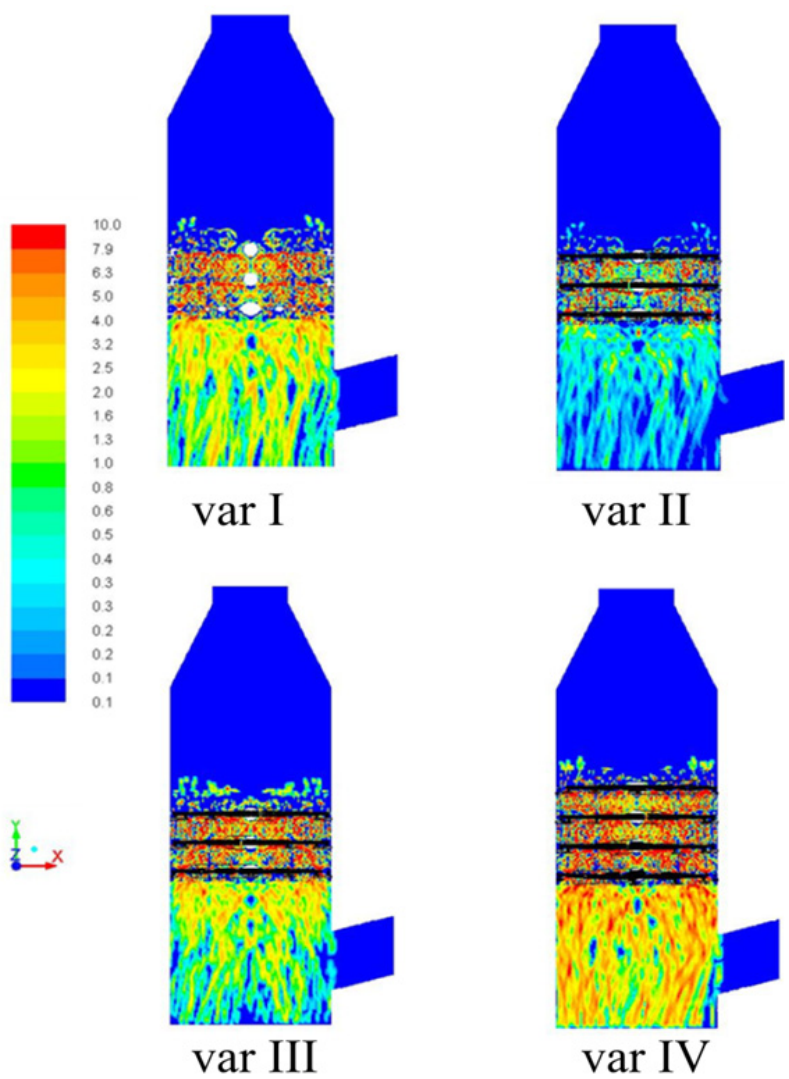

Figure 8 Concentration of suspension $\left(\mathrm{kg} / \mathrm{m}^{3}\right)$ - plane $\mathrm{z} 0$. 


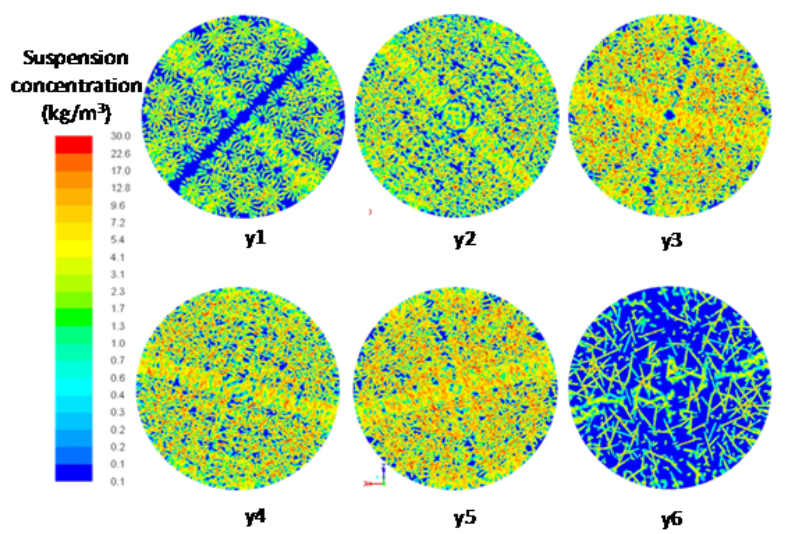

Figure 9.Var. I. - concentration of suspension.

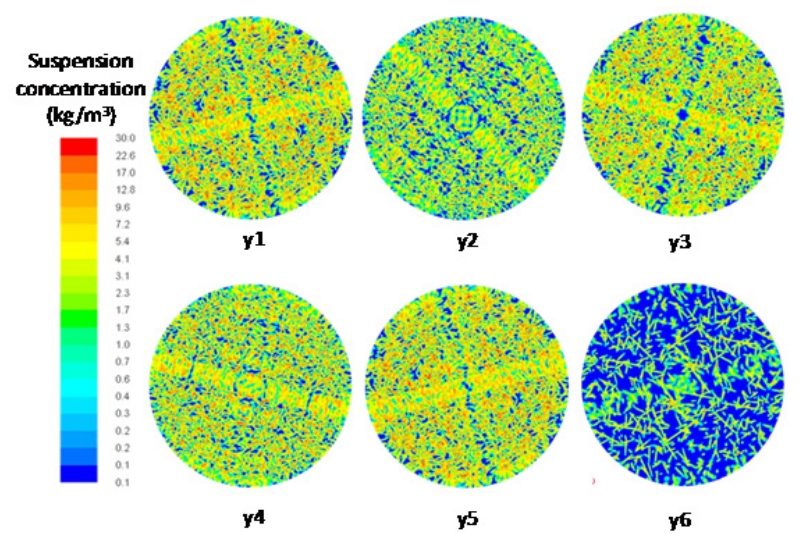

Figure 10. Var. III. - concentration of suspension.

\section{Conclusion}

Numerical simulations performed in this work do not reflect the kinetics of chemical reactions. From the results it is not possible to evaluate the concentration of $\mathrm{SO}_{2}$ at the outlet of the scrubber. The model gives detailed information about the distribution of the concentration of suspension in the reaction zone and the velocity and temperature field in the flue gas. The model also can relatively accurately predict the effect of particle size on the concentration of suspension in scrubber.

It can be assumed that exist a certain range of the average concentration of suspension, which is optimal for the desulphurisation process. The changes in the scrubber should lead to increase the percentage of volume flow rate of flue gas in the "optimum zone" As stated above, the kinetics of chemical reactions is affected by other parameters, but they can't be included in this model. The most important are effect of temperature on reaction rate, the effect of particle size on reaction rate, time course of concentration of reactants in suspension and distribution of reactants in suspension. Preparing a model that could also affect these parameters is possible. The correct setting depends on the quality of the input data, especially models and constants for the description of chemical reactions.
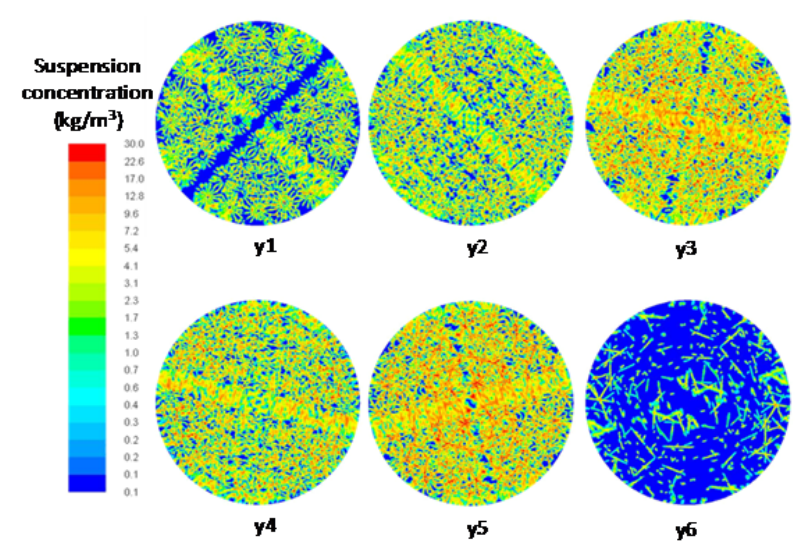

$\mathbf{y}$

Figure 11. Var. II. - concentration of suspension.

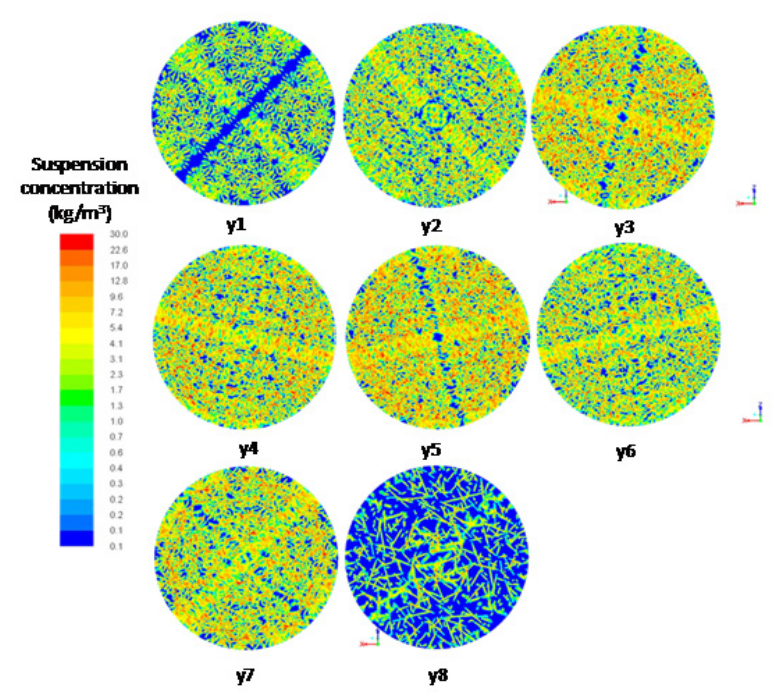

Figure 12. Var. IV. - concentration of suspension.

Obtaining these constants and their implementation into the computational model should be explored further by experiments.

\section{Acknowledgement}

Author gratefully acknowledges financial support by Czech Technological Agency under the project TACR TA04021338 and SGS 21000.

\section{References}

1. Ansys Inc., Fluent User's Guide Release 14.5, 2012

2. A. Bes, Dynamic Process Simulation of Limestone Calcination in Normal Shaft Kilns, Fakultät für Verfahrens- und Systemtechnik der Otto-von-Guericke-Universität Magdeburg, 2005

3. J. Xu et al, Numerical Study of the Effects of Flue-Gas Inlet Type for the Flue-Gas Desulfurization Wet Scrubber, CHEMICAL ENGINEERING TRANSACTIONS Volume 21, 2010 